\title{
Aplicação das ferramentas de abordagem familiar em uma ESF de um município de Minas Gerais: um relato de caso
}

\author{
Application of family approach tools in an ESF in a municipality in Minas Gerais: a case \\ report
}

\section{Aplicación de herramientas de aproximación familiar en un FSE en un municipio de Minas Gerais: informe de un caso}

Ludmila Cotrim Fagundes $^{1 *}$, André Augusto Dias Silveira ${ }^{1}$, Alexandre Vitor Dias Silveira ${ }^{2}$, Daniela Cotrim Fagundes ${ }^{3}$, Lucas Lopes Fagundes ${ }^{2}$, Gabriela Lopes Fagundes ${ }^{2}$, Ana Laura Oliveira Santos Dias Guimarães ${ }^{2}$, Antonio Silveira de Sá ${ }^{1}$, Ariadna Janice Drumond Morais¹.

\section{RESUMO}

Objetivo: Descrever a utilização da abordagem familiar no atendimento à saúde primária. Detalhamento do caso: Trata- se de um relato de caso desenvolvido no âmbito de uma ESF (Estratégia Saúde da Família) de um município de Minas Gerais, sobre a utilização de ferramentas próprias para a Abordagem Familiar: Genograma, F.I.R.O., P.R.A.C.T.I.C.E., Ciclo de Vida e Ecomapa. Entrevistado A.F.S., 53 anos, hígido, casado, desempregado e pai de quatro filhos, o segundo Autista, a quarta portadora de Síndrome de Down e esposa depressiva. $\mathrm{Na}$ aplicação das ferramentas nota-se que este é o nono filho de um casal cuja mãe é hígida e o pai é hipertenso. Apesar das dificuldades encontradas nas relações familiares, principalmente com o filho, tem bastante reconforto na religião. Considerações finais: A família é caracterizada por um conjunto de crenças, valores e normas, sendo fundamental que o profissional da saúde a compreenda como um elemento de prática diagnóstica e terapêutica. Sendo sua abordagem durante atendimento médico na atenção primária um aspecto fundamental no entendimento do indivíduo como um todo. Estudo de Família permite entender a dinâmica familiar, propondo soluções mais aplicáveis a realidade, entretanto, precisa-se de mais estudos dessas ferramentas e mais aderência dos médicos de família e comunidade.

Palavras-chave: Assistência integral à saúde, Relações familiares, Medicina de família e comunidade.

\begin{abstract}
Objective: To describe the use of the family approach in primary health care. Details of the case: This is a case report developed within the scope of an ESF (Family Health Strategy) of a municipality in Minas Gerais, on the use of own tools for the Family Approach: Genogram, FIRO, PRACTICE, Cycle of Life and Ecomap. Interviewed A.F.S., 53, healthy, married, unemployed and father of four children, the second Autistic, the fourth carrier of Down Syndrome and a depressive wife. In the application of the tools, it is noted that this is the ninth child of a couple whose mother is healthy and the father is hypertensive. Despite the difficulties found in family relationships, especially with the son, he has a lot of comfort in religion. Final considerations: The family is characterized by a set of beliefs, values and norms, being essential that the health professional understands it as an element of diagnostic and therapeutic practice. Its approach during medical care in primary care is a fundamental aspect in the understanding of the individual as a whole. Family Study allows understanding the family dynamics, proposing solutions that are more applicable to reality, however, more studies of these tools and more adherence by family and community doctors are needed.
\end{abstract}

Keywords: Comprehensive health care, Family relations, Family practice.

1 Universidade Estadual de Montes Claros, Montes Claros - MG. *E-mail: ludmilacotrimfagundes@gmail.com

2 Centro Universitário FIPMoc, Montes Claros - MG.

${ }_{3}^{3}$ Universidade Federal dos Vales do Jequitinhonha e Mucuri, Diamantina - MG. 


\section{RESUMEN}

Objetivo: Describir el uso del enfoque familiar en la atención primaria de salud. Detalle del caso: Se trata de un caso clínico desarrollado en el ámbito de una ESF (Estrategia de Salud de la Familia) de un municipio de Minas Gerais, sobre el uso de herramientas propias para el Enfoque Familia: Genograma, FIRO, PRÁCTICA, Ciclo de Life y Ecomap. Entrevistado a A.F.S., 53 años, sano, casado, desempleado y padre de cuatro hijos, el segundo autista, el cuarto portador del síndrome de Down y una esposa depresiva. En la aplicación de las herramientas, se observa que este es el noveno hijo de una pareja cuya madre está sana y el padre hipertenso. A pesar de las dificultades encontradas en las relaciones familiares, especialmente con el hijo, él tiene mucho consuelo en la religión. Consideraciones finales: La familia se caracteriza por un conjunto de creencias, valores y normas, siendo fundamental que el profesional de la salud la comprenda como un elemento de la práctica diagnóstica y terapéutica. Su abordaje durante la atención médica en atención primaria es un aspecto fundamental en la comprensión del individuo en su conjunto. El Estudio de Familia permite comprender la dinámica familiar, proponiendo soluciones más aplicables a la realidad, sin embargo, se necesitan más estudios de estas herramientas y más adherencia por parte de los médicos de familia y comunitarios.

Palabras clave: Atención integral de salud, Relaciones familiares, Medicina familiar y comunitaria.

\section{INTRODUÇÃO}

A saúde compreende aspectos biológicos, físicos, psicológicos, socioeconômicos e culturais, além da oportunidade de acesso aos serviços de promoção, proteção e recuperação (DUCAN BB, et al., 2013). Por ser a família caracterizada por um conjunto de crenças, valores e normas, é fundamental que o profissional da saúde a compreenda como um elemento de prática diagnóstica e terapêutica ampla (CHAPADEIRO CA, et al., 2011; BRASIL MS, 2012). Sendo o foco de Atenção Primária à Saúde (APS) contribuir para modificação do modelo biomédico de saúde na Estratégia de Saúde da Família (ESF) (SILVA MCLSR, et al., 2011).

A ESF tem por objetivo melhorar Atenção Básica à Saúde no país, em consonância com os preceitos, princípios e diretrizes do Sistema Único de Saúde (SUS), promovendo dessa forma a ampliação da democratização da saúde no Brasil. Ela pressupõe também a integralidade do cuidado sobre a população que reside na área de abrangência de suas unidades de saúde (FERTONANI HP, et al., 2015).

A unidade de saúde da família atua em área específica, dividida em micro áreas, e sob uma populaçãoalvo, que deve ser observada com objetivo de se fazer o levantamento de dados relacionados à qualidade de vida da comunidade assistida.

Alguns critérios devem ser avaliados, como doenças mais comuns entre os moradores, segurança, meios de transporte mais utilizados, áreas de lazer, saneamento básico, escolaridade, nível socioeconômico e relações familiares, a fim de melhorar o bem-estar e a saúde da comunidade, respeitando as particularidades da região observada (FIGUEIREDO EN, 2012).

Nesse ínterim, criou-se as ferramentas de trabalho com famílias, tecnologias relacionais que visam estimular a compreensão da estrutura familiar e estreitar a relação do profissional com o paciente (LACERDA MKS, et al., 2017).

Entre elas estão: o Genograma, que ilustra a estrutura, forma, relacionamentos e doenças presentes na família estudada; o Ciclo de Vida Familiar, que possibilita o posicionamento da família entre os diferentes estágios de vida; o F.I.R.O. (Orientações Fundamentais nas Relações Interpessoais), que avalia a vivência cotidiana e sentimentos dos familiares; o P.R.A.C.T.I.C.E. (Present Problem; Roles and Structure; Affect; Communication; Time in the family life cycle; Illness in family past and present; Coping with stress; Ecology), que permite avaliar a família sob as perspectivas do que cada letra da sigla sugere; e o Ecomapa, que ilustra graficamente as relações da família com o meio que a circunda (BRANTE ARSD, et al., 2016; SANTOS KKF, et al., 2015; DIAS LC e LOPES JMC, 2015).

Estas são ferramentas que podem ser aplicadas nas consultas médicas através de perguntas ao paciente, visando a compreensão do indivíduo como um todo, para auxiliar no processo de interação médico-paciente e ajudar a entender como algumas situações familiares podem refletir o processo de adoecimento da pessoa. Portanto, o objetivo deste trabalho é descrever um relato de caso sobre a aplicação dessas ferramentas durante atendimento médico a uma família em uma ESF de um munícipio de Minas Gerais. 


\section{DETALHAMENTO DO CASO}

O presente trabalho trata-se de um relato de caso desenvolvido no âmbito de uma ESF de um munícipio de Minas Gerais, durante consulta médica, no dia 12 de novembro de 2017, sobre a utilização de ferramentas próprias para a Abordagem Familiar. Paciente: A.F.S., 53 anos, hígido, casado e pai de quatro filhos. Aplicouse as seguintes ferramentas para o estudo da família: genograma, ciclo de vida familiar, F.I.R.O, P.R.A.T.I.C.E. e Ecomapa. O genograma evidencia o paciente A.F.S., nascido em 1964, casado, com relação de amor com a esposa que possui transtorno depressivo. Desse casamento de 17 anos nasceram 4 filhos: 0 primeiro homem de 16 anos, hígido; o segundo de 13 anos com autismo, com quem o pai possui uma relação hostil; o terceiro uma menina de 10 anos, hígida; e o último uma criança, do sexo feminino, de 4 anos com Síndrome de Down, com quem o pai possui uma relação de muito amor. A.F.S. é o nono filho do casamento harmonioso de seus pais e o sétimo homem, seu segundo irmão possui doença mental e sua irmã mais nova, Síndrome de Down. Sua mãe de 83 anos é hígida e seu pai de 92 anos é hipertenso. A esposa de A.F.S. é a quarta filha de seis filhos, fruto do casamento harmonioso de seus pais. O sogro de A.F.S. possui cegueira unilateral, sua sogra é hipertensa, seu cunhado mais velho está em recuperação de um Acidente Vascular Encefálico (AVE). Seu segundo cunhado possui Doença de Chagas, o terceiro faleceu em 1999 devido a esta mesma doença; e as outras duas cunhadas hígidas (Figura 1).

Figura 1 - Genograma do paciente AFS.
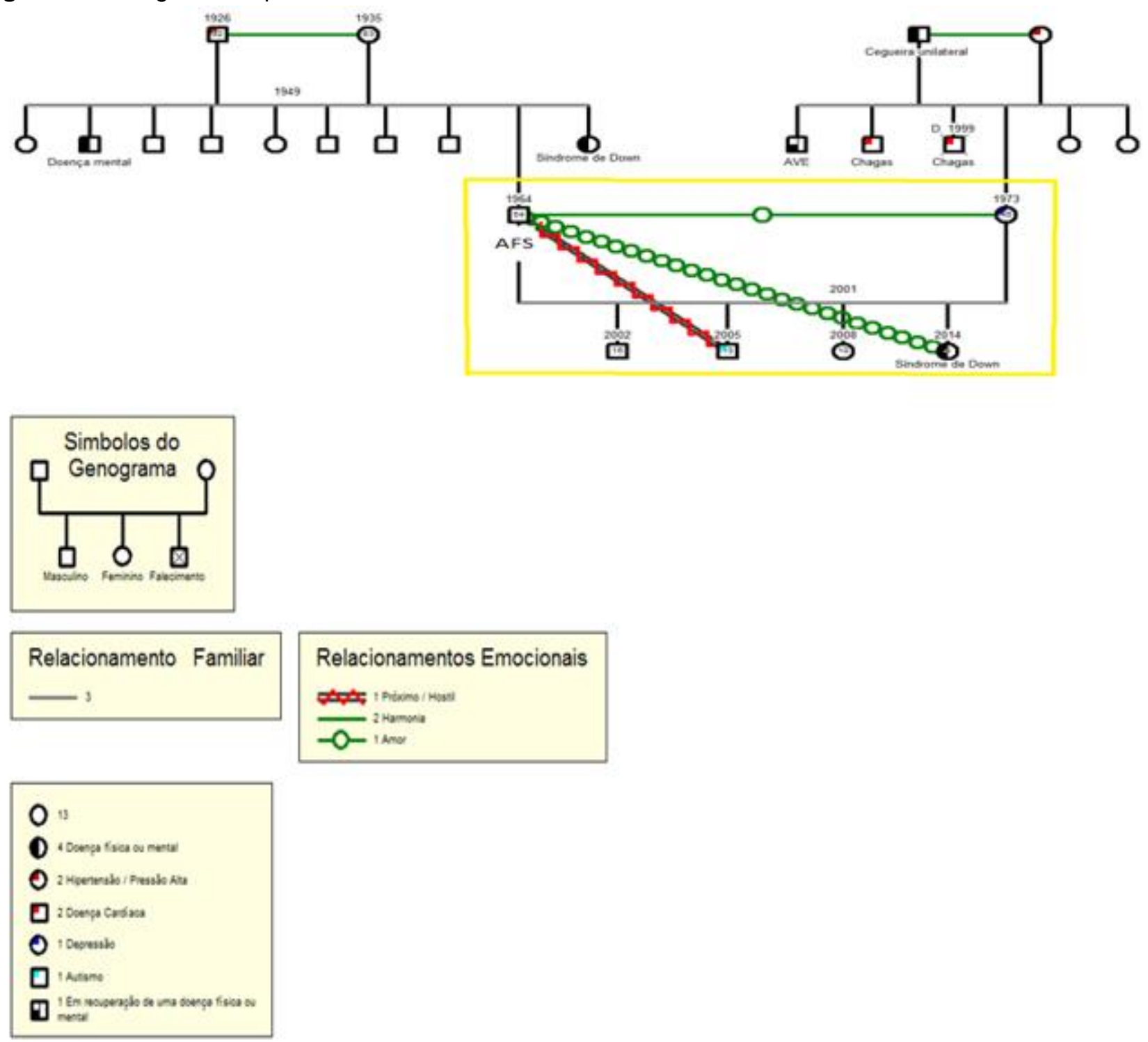

Fonte: Fagundes LC, et al., 2020. 
O Ciclo de vida familiar pode ser dividido em oito fases de desenvolvimento, sendo a primeira delas o início da vida de um casal como família. O segundo estágio se refere ao momento em que o casal possui filhos pequenos. No terceiro estágio as crianças estão em fase pré-escolar. Estágio IV os filhos se encontram em estágio escolar.

No quinto estágio possuem filhos adolescentes e no Estágio VI as famílias são o centro de partida. Já no Estágio VII o casal está na meia idade e no Estágio VIII, na terceira idade. Ciclo de Vida da família analisada - Estágio IV - famílias com filhos em idade escolar. O F.I.R.O. (Orientações Fundamentais nas Relações Interpessoais) analisa os familiares quanto aos sentimentos e seu convívio.

\section{F.I.R.O.}

Inclusão: a estrutura da família é composta por 4 filhos e esposa vivendo na mesma casa. Mulher dona de casa e marido ex-açougueiro, hoje com trabalhos provisórios e incertos. Em relação a conectividade, família unida, religiosa, em harmonia e que tentam juntos superar os desafios da depressão da esposa, desemprego paterno, um filho autista e filha com Síndrome de Down. Quanto ao compartilhamento, compartilham medos e alegrias entre os membros. Pai, única fonte de renda familiar, parou de trabalhar como açougueiro e passou a fazer "bicos", porque assim passa mais tempo em casa com os filhos e mulher, esta que precisa de apoio devido a depressão e dificuldades para cuidar do filho autista e filha com Síndrome de Down.

Controle: não há membro dominante. A análise do controle reativo, filho com autismo apresenta atitudes violentas e hostis com frequência, mas os pais conseguem controlar e não desaminam nem desapontam, acreditando que em tudo há um proposito divino. Família marcada pela cooperação entre os membros nas atividades domésticas; filhos mais velhos cuidando e compreensivos com o filho autista e filha com Síndrome de Down. Decisões tomadas com envolvimento de todos.

Intimidade: A.F.S. sente-se confortável em compartilhar seus sentimentos com a família, vive em grande parceria com a esposa e está satisfeito com as relações familiares do lar. No modelo P.R.A.C.T.I.C.E. cada letra indica uma questão a ser investigada: Problem; Roles and structure; Affect; Communication; Time in life; Illness in Family; Coping with stress; Environment or Ecology.

\section{P.R.A.C.T.I.C.E.}

Problema apresentado (Problem): Depressão da esposa, filho autista e filha com Síndrome de Down. Esta apresenta quadros repetitivos de internações por pneumonia e possui problema na adenoide. Filho autista requer acompanhamento periódico com neuropediatra, o que o preocupa por dificuldades financeiras para consultas particulares e demora do atendimento público.Papéis e estrutura (Roles and structure): Nega conflitos, Bolsa Família e A.F.S. são única fonte de renda, este através de trabalhos provisórios como açougueiro, porque não pode ter empregos fixos pela necessidade de estar sempre em casa prestando apoio a esposa e aos filhos com problemas. Mulher dona de casa, mas marido também se encontra colaborativo.

Afeto (Affect): Família bastante afetuosa. Informam que após nascimento da última filha o afeto aumentou ainda mais, apesar dos problemas financeiros, relata que esta filha é mais afetuosa e presente na vida dos pais, preenchendo o vazio da mãe e acreditando ser um anjo enviado por Deus. Comunicação (Communication): Família próxima e comunicação presente e não-conflitante entre os membros.

Tempo no ciclo de vida (Time in life): Família que vivenciou fases difíceis desde a aprender lidar com filho autista, depressão materna ou aumento dos gastos com saúde concomitante a diminuição da renda paterna, entretanto, muito religiosos e encontram nisso conforto e entendimento para superar tudo juntos. Doenças na família, passadas e presentes (Illness in family): Sem histórias de enfermidades passadas, acreditam que a doença é um ensinamento de Deus para tornarem melhores. Gostam do serviço de saúde que tem acesso, principalmente a atenção primária os ajudam bastante.

Lidando com o estresse (Coping with stress): Calmos e acreditam que os filhos com problemas puderam treinar para serem pessoas melhores e mais cautelosas. Meio ambiente ou Ecologia (Environment or Ecology): A.F.S. tem nos filhos, esposa e religião a base de vida. Encontra-se apoiado pelo sistema de saúde, apesar de reclamar da demora. Não confia nos vizinhos. Pôde também contar com os pais ou sogros sempre que precisou, principalmente em se tratando da questão financeira. 
E por fim, o Ecomapa, o qual evidencia as relações da família com outras instituições, esclarecendo o equilíbrio entre elas. No Ecomapa, a família de A.F.S. possui relação de grande apoio mútuo com a igreja; possui uma relação superficial com os vizinhos de residência do bairro; relação mútua, mas não muito profunda, com o colégio, onde seu filho mais velho estuda. A família recebe apoio significativo da escola, onde estuda e estudaram os filhos em idade de ensino primário.

O apoio da APAE (Associação de Pais e Amigos dos Excepcionais) para a família de A.F.S. é de grande repercussão positiva na família, pelo bom acolhimento da filha mais nova, a qual possui Síndrome de Down. Possuem relação com a ESF Vila Sion e Supermercado parecida, de bom apoio fornecido por essas instituições e que, mesmo com pouca oportunidade logística, tentam cooperar com elas.

A família oferece apoio ao Hospital através da participação de ações comunitárias e doações, mas sem necessitar muito de buscar apoio dessa instituição, por suas requisições serem bem cumpridas por parte da ESF Vila Sion. Com relação aos parentes, a família está sempre recebendo e oferecendo ajuda quando oportuno, mas não são tão próximos, por vezes em outra cidade (Figura 2).

Figura 2 - Ecomapa da família de AFS.

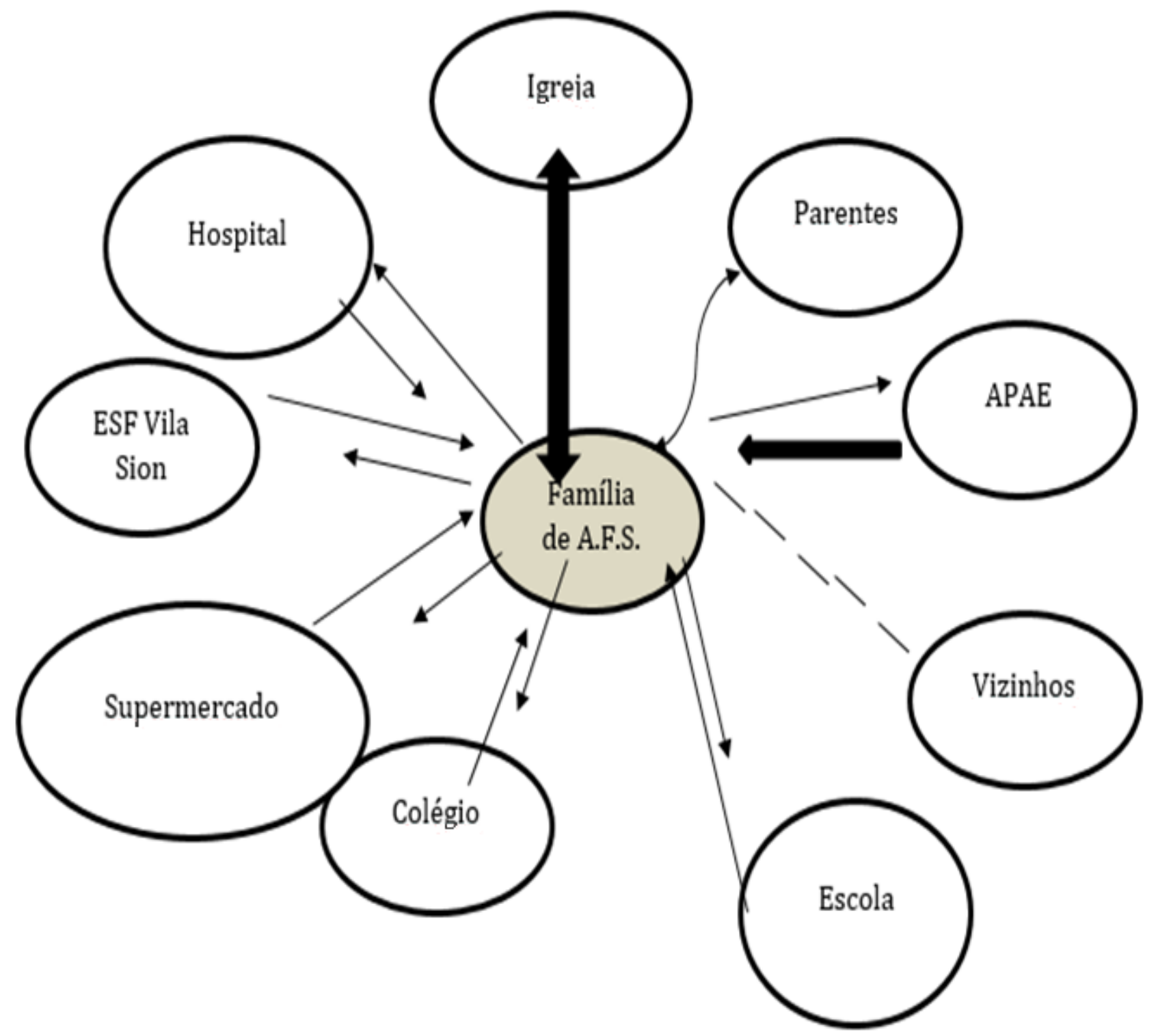

Fonte: Fagundes LC, et al., 2020. 


\section{DISCUSSÃO}

Através dos instrumentos de abordagem familiar utilizados na consulta da atenção primária é possível estreitar o relacionamento do profissional de saúde com o paciente, assim como promover maior compreensão do funcionamento do indivíduo e seu relacionamento com sua família e comunidade (MOREIRA LT, et al., 2018). Em sintonia com isso, a aplicação das ferramentas de abordagem familiar no âmbito da ESF em questão pode agregar bastante na relação médico paciente nessa atenção básica de saúde.

Na família em questão, através do Genograma, depreende-se como A.F.S. relaciona-se com seus filhos, a sua situação conjugal, patologias presentes ao longo de três gerações e como as patologias em questão podem influenciar os seus relacionamentos, como é o caso do autismo presente em seu segundo filho, uma possível causa agravante do seu relacionamento conturbado com o garoto.

Em outros casos, como o estudo de Oliveira VCA, et al. (2017), a doença pode funcionar como um fator de aproximação entre membros da família e até gerar certo grau de dominância como ocorreu com a paciente índice que apresentou quadro de déficit cognitivo e câncer bucal.

Em sua experiência, Brante ARSD et al. (2016), descreve uma família em estágio VI do Ciclo de Vida, em que se encontra em estágio de ninho vazio, com a partida das crianças do núcleo familiar. Já a família de A.F.S. encontra-se na fase IV, caracterizada por crianças em fase escolar.

Essa fase está associada a várias dificuldades no contexto financeiro, por possuir membros que não contribuem na renda total familiar; e no contexto educacional, principalmente em se tratando de crianças com autismo e Síndrome de Down, como no caso em questão.

Nesse contexto de compreensão das alterações no ciclo de vida da família, o F.I.R.O. avalia os familiares sob a perspectiva de suas relações e sentimentos associados a patologias presentes (SANTOS JAD, et al., 2016). No caso de A.F.S., a inclusão é positiva, com boa conectividade entre os membros; não existe um membro dominante, todos os assuntos familiares são discutidos em comum acordo entre os envolvidos, principalmente devido a boa relação de intimidade familiar ao compartilhar anseios e desejos.

$\mathrm{Na}$ avaliação do P.R.A.C.T.I.C.E. o "P" da família em questão refere-se a problemas relacionados ao acesso a saúde pelos membros. $O$ "R" refere-se a estrutura familiar e suas tarefas, a qual se mostra apoiadora e sem problemas relatados.

O "A" refere-se ao afeto, sendo muito positivo entre A.F.S. e a filha mais nova, o paciente foco do caso baseia todo o seu afeto em sua religiosidade e crença cristã. $O$ "C" refere-se a boa comunicação entre os membros.

O "T" refere-se a diferentes fases do ciclo de vida, pelas quais a família passou por dificuldade e conseguiu superar com apoio da religião. O "I" diz respeito a doenças passadas e da família, que no caso em questão não há nenhuma além das mencionadas.

$\mathrm{O}$ "C" indica como a família lida com o estresse, na família do caso todos lidam bem com as adversidades e creem que elas ajudam na evolução pessoal de cada um. O "E" discorre acerca do envolvimento ecológico, a família mantém um ambiente harmonioso e de parceria.

O Ecomapa correlaciona os familiares com o meio ambiente e destaca seus vínculos com os recursos da comunidade (SILVEIRA BJ, et al., 2018). A família de A.F.S. possui relação próxima e mútua com a igreja, relação mútua, mas não profunda com a escola de seu filho, recebe apoio da APAE e relação superficial com vizinhos. Rosário MS, et al. (2019), em seu estudo encontra uma família que, assim como a de A.F.S., possui uma relação estreita de apoio com a igreja e uma relação superficial com os vizinhos.

As ferramentas abordagem familiar são importantes na avaliação holística do indivíduo. O Estudo de Família permite entender a dinâmica familiar, propondo soluções mais aplicáveis a realidade, assim, o profissional de saúde é capaz de identificar fatores de moradia, higiene, relacionamentos, aspectos socioeconômicos, culturais e estilo de vida envolventes no problema. Entretanto, precisa-se de mais estudos dessas ferramentas e mais aderência dos médicos de família e comunidade. 


\section{REFERÊNCIAS}

1. BRANTE ARSD, et al. Abordagem Familiar: aplicação de ferramentas a uma família do município de Montes Claros/MG. Rev Bras Med Fam Comunidade, 2016; 11(38): 1-9.

2. BRASIL MS. Caderno de atenção domiciliar. 2ed. Ministério da Saúde, 2012; 207p.

3. CHAPADEIRO CA, et al. A família como foco da atenção primária à saúde. Nescon/UFMG, 2011; 96p.

4. DIAS LC, LOPES JMC. Abordagem familiar na atenção domiciliar [material instrucional]. Porto Alegre: UFSC, 2015; $82 p$.

5. DUNCAN BB, et al. Medicina ambulatorial: condutas de atenção primária baseadas em evidências. 4aㅡ Ed. Porto Alegre: Artmed, 2013; 1976p.

6. FERTONANI HP, et al. Modelo assistencial em saúde: conceitos e desafios para a atenção básica brasileira. Ciênc. saúde coletiva, 2015; 20(6): 1869-78.

7. FIGUEIREDO EN. A Estratégia Saúde da Família na Atenção Básica do SUS. São Paulo: Universidade Federal de São Paulo - UNA-SUS/UNIFESP, 2012; 12p.

8. LACERDA MKS, et al. Ferramentas de Abordagem Familiar: estudo de uma família cadastrada em uma equipe de estratégia saúde da família em Montes Claros, Minas Gerais, Brasil. Revista de Iniciação Científica da Universidade Vale do Rio Verde, 2017; 7(1): 25-34.

9. MOREIRA LT, et al. Abordagem familiar: quando, como e porquê? Um caso prático. Rev Port Med Geral Fam, 2018; 34(4): 229-236.

10. OLIVEIRA VCA, et al. Aplicação de ferramentas de abordagem familiar na estratégia saúde da família. Rev Bras Promoç. Saúde, 2017; 30(4): 1-8.

11. ROSÁRIO MS, et al. Aplicação de Ferramentas de Abordagem Familiar no âmbito Estratégia Saúde da Família: um relato de caso. Revista Eletrônica Acervo Saúde, 2019; Sup25(783): 1-9.

12. SANTOS JAD, et al. Ferramenta de abordagem familiar na atenção básica: um relato de caso. J Health Sci Inst., 2016; 34(4): 249-252.

13. SANTOS KKF, et al. Ferramentas de abordagem familiar: uma experiência do cuidado multiprofissional no âmbito da estratégia saúde da família. Revista da Universidade Vale do Rio Verde, 2015; 13(2): 377-387.

14. SILVA MCLSR, et al. A abordagem à família na Estratégia Saúde da Família: uma revisão integrativa da literatura. Rev Esc Enferm USP, 2011; 45(5): 1250-1255.

15. SILVEIRA BJ, et al. Aplicação de ferramentas de abordagem familiar na atenção primária: um relato de caso. Revista Eletrônica Acervo Saúde, 2018; 15: S2002-S2008. 